Article

\title{
Developing Stress Management Programs in a Public Primary Healthcare Institution: Should We Consider Health Workers' Sociodemographic Groups?
}

\author{
Daiva Dudutienė *(1), Audronè Juodaitè-Račkauskienė and Rimantas Stukas \\ Department of Public Health, The Institute of Health Sciences, The Faculty of Medicine, Vilnius University, \\ LT-03101 Vilnius, Lithuania \\ * Correspondence: daiva.dudute@gmail.com; Tel.: +37-06-8761-849
}

Received: 11 March 2020; Accepted: 1 April 2020; Published: 3 April 2020

check for updates

\begin{abstract}
Background and Objectives: An essential part of occupational stress management is identifying target groups and developing a wellbeing program that tailors interventions to the specific needs of the target groups. This study aims to explore whether psychosocial risk determinants and organizational intervention objects differ across employees' groups based on sociodemographic factors in a Lithuanian public primary healthcare institution. Methods: All 690 health workers of the institution were invited to participate (response rate 68\%) in a cross-sectional survey between February and March 2017. The questionnaire contained items related to sociodemographic factors (gender, age, job seniority, education, and occupation), 14 psychosocial risk determinants, and 10 organisational intervention objects. Results: The results of the study showed that differences by gender were not statistically significant except for one organisational intervention object (work-life balance). Only a few organisational intervention objects (justice of reward, matching to the job demand, and variety of tasks) had mean rank scores differing statistically across age and job seniority groups. Five organisational intervention objects (work-life balance, variety of tasks, communication, manager feedback, and stress management training) had mean rank scores differing statistically across education groups, and all organisational intervention objects (except stress management training) had mean rank scores differing statistically across occupational groups. Regarding psychosocial risk determinants, excessive work pace had mean rank scores differing statistically across age and job seniority groups. Four (overtime, unclear role, conflicting roles, and being under-skilled) and six psychosocial risk determinants (work overload, overtime, tight deadlines, unclear role, being under-skilled, and responsibility) had mean scores differing statistically across education and occupational groups, respectively. Statistical significance was considered with $p$-value $<0.05$ and 95\% confidence interval. Conclusions: The findings showed that different psychosocial risk determinants and organizational interventional objects were emphasized by different sociodemographic groups in the institution, but they did not impact groups in the same measure. Therefore, it is crucial to start by determining the risk group's specific needs before developing and implementing stress management programs.
\end{abstract}

Keywords: occupational safety and health; job stress; psychosocial risks; organisational interventions; health professionals or health workers; wellbeing at work

\section{Introduction}

Patients have high expectations of the speed and quality of healthcare, so demand and time pressures are increasing. The digital transformation of health services and fast changing work environment are additional challenges for health workers. Problems observed in a number of 
healthcare institutions include increased staff shortage, staff turnover, sickness absenteeism, high workload, and work demands. In addition, the health workforce itself is ageing and there are problems of retention due to demanding working conditions and relatively low pay in some countries. Thus, it comes as no surprise that the health workforce is an occupational group whose wellbeing and job performance are adversely affected by job stressors. Many researchers have investigated the relationship between job stressors and job performance. Many studies also showed consistently negative relationships between job stressors and job performance [1,2]. Policies that decrease job stress have the potential to improve the health of employees and to reduce the burden of absenteeism due to illness $[3,4]$.

However, there is no simple solution to managing job stressors and to improving employees' health and wellbeing at work. At an EU level, it is the employers' obligation to improve the working environment by dealing with all types of risk, including psychosocial risks [5]. Over the last century, many studies have been conducted on how to better implement the welfare policy at work. Numerous qualitative and quantitative studies have explored how to improve psychosocial work environment in many countries. A systematic review of the job stress intervention evaluation literature in 1990-2005 [6] has discovered that organisationally directed interventions are more beneficial than individually directed interventions. Some studies have documented the positive effects of the organisational stress management interventions [7-9], while others have documented little, no, or negative effects of these interventions $[10,11]$. Some researchers have pointed out that variety of factors-individual personality, job profile, workplace culture, standards, and/or regulations in different areas-may lead to different outcomes [12,13]. Debate continues about the best approaches for psychosocial risk management at work. In the last century, the majority of studies aimed at finding a one-size-fits-all approach to the design of wellbeing at work [14]. There is still a gap between theoretical knowledge on psychosocial risk management and respective practical applications. Therefore, researches have recently turned to analysing preconditions and to examining the role of intervention by the organisation to the individuals within it $[15,16]$. Results of a meta-analysis of person-job, person-organisation, person-group, and person-supervisor fit "provide strong evidence for the importance of multiple types of fit for work-related attitudes and behaviours" [17]. A multiple approach may enable an employer to make "an assessment of its relative position (compared to "the average employee" and to specific norm-scores of the branch) and to make internal comparisons between departments or groups in the organisation (on the basis of age, gender, blue versus white collar, and so on)" [14]. This study aims to contribute to this growing area of research by exploring the importance of the sociodemographic factors in developing a psychosocial risk management strategy to improve employee wellbeing in the organisation. Thus, the study was designed to assess the differences of groups' attitudes (on the basis of gender, age, job seniority, education, and occupational status) toward psychosocial risk determinants and organizational intervention objects in order to obtain information that is not captured by the dominant evaluation paradigm [18,19].

\section{Materials and Methods}

\subsection{Study Sample and Design}

This study is a cross-sectional study designed to examine health workers' attitudes toward the psychosocial risk determinants and organizational intervention objects using a complex quantitative tool based on sociodemographic factors. All data was collected by paper questionnaires from February to March 2017. The sample consisted of 467 health workers employed in one of the largest primary healthcare institutions (the eight healthcare institutions were merged into one in 2002) in Lithuania. The institution employed 690 health workers in 2017. All health workers were invited to participate in the study. At the start of the study, health workers were provided with information about the study and 690 paper questionnaires were distributed; 468 questionnaires were returned compiled, one of 
which was damaged; 467 questionnaires (response rate 68\%) were suitable for the analysis of the research results.

\subsection{Ethical Considerations}

Participation in the survey was voluntary with guaranteed anonymity and confidentiality under Lithuanian law which does not require ethical approval for this type of study. The study was authorised by the administration of the institution.

\subsection{Instruments/Measures}

The self-administrated questionnaire was used as an instrument for data collection. The instrument contained items adopted from an established questionnaire that was used for complex stress management study at Lithuanian automation and electrotechnical companies [20]. The questionnaire consisted of 67 items, divided into three parts related to psychosocial risk determinants, organisational intervention objects, and sociodemographic factors; Cronbach's alpha was 0.89 for the general scale. However, before using the instrument in this study, it was subjected to a pilot testing where 50 questionnaires were administered to 50 health workers in the institution. After the pilot study and a discussion with the administration of the institution, an abridged and adapted to health work version of the validated instrument was used:

-Fourteen items for psychosocial risk diagnosis: hazardous working conditions; work overload; excessive work pace; overtime; tight deadlines; unclear role; conflicting roles; being under-skilled for a job; responsibility for decision making and actions; lack of control over work pace; lack of control over work method; and interpersonal relationships (harassment, conflicts, and tension). Cronbach's alpha $=0.702 ;$

-Twenty-seven items for organisational intervention object ascertainment: social support ( 5 items; Cronbach's alpha $=0.704)$, organisational support $(4$ items; Cronbach's alpha $=0.708)$, participation in decision making ( 3 items; Cronbach's alpha $=0.66$ ); communication ( 4 items; Cronbach's alpha $=0.728$ ); justice of reward ( 2 items; Cronbach's alpha $=0.756)$; manager feedback ( 5 item; Cronbach's alpha $=0.738)$; stress management training $(1$ item); work-life balance ( 1 item); skills/abilities matching to the job demands (1 item); and variety of tasks (1 item);

-Five groups on the basis of sociodemographic factors: gender (male, female), age (coded on four levels: $\leq 30,31-40,41-50$, and $>50$ years), job seniority (coded on four levels: $\leq 3,3.01-5,5.01-10$, and $>10$ working years), educational level (coded on three levels: university degree, higher school/college degree, and other degree), and occupational status (coded on four levels: heads of units, doctors, nurses, and other health workers (ergotherapists, masseurs, etc.)).

In total, the questionnaire consisted of 46 items, which were rated on a 5-point frequency scale ranging from 1 (never or strongly disagree) to 5 (always or strongly agree). Prior to analyses, some items of the questionnaire were reversed so that higher scores showed greater negative impact of psychosocial risk determinant and more relevant to organisational intervention object.

\subsection{Statistical Analysis}

Data were analyzed using the statistical software package IBM SPSS Statistics (Vilnius University, Vilnius, Lithuania). In all analyses, statistical significance was considered with $p$-value $<0.05$ and $95 \%$ confidence interval (CI). A descriptive analysis was carried out to examine the sociodemographic groups of employees in the institution. Nonparametric tests for comparisons of the groups (the Mann-Whitney test for comparisons of two groups and the Kruskal-Wallis test for comparisons of more than two groups) were used then.

\section{Results}

Table 1 describes sociodemographic groups in the institution. As is the case for many Lithuanian healthcare institutions, the results showed a predominance of women $(94.9 \%)$. Health workers were 
aged 22 to 73 years old. Almost half of health workers (47.9\%) were over 50 years of age. More than half of all health workers (52.9\%) had university degrees, 38.5\% of health workers had higher school degrees, and $8.6 \%$ of health workers had other levels of education. The majority of health workers were nurses $(43.9 \%)$, followed by doctors $(28.3 \%)$, other health workers $(21.6 \%)$, and heads of units $(6.2 \%)$. Health workers' seniority ranges are from a few months to 48 years; 350 of the health workers worked over 10 years $(76.1 \%)$.

Table 1. Groups on the basis of sociodemographic factors.

\begin{tabular}{|c|c|c|c|}
\hline \multicolumn{2}{|c|}{ Groups } & $N$ & $\%$ \\
\hline \multirow{3}{*}{ Gender } & Male & 24 & 5.1 \\
\hline & Female & 443 & 94.9 \\
\hline & Total & 467 & 100 \\
\hline \multirow{5}{*}{ Age } & $\leq 30$ & 48 & 10.5 \\
\hline & $31-40$ & 68 & 14.8 \\
\hline & $41-50$ & 123 & 26.8 \\
\hline & $>50$ & 220 & 47.9 \\
\hline & Total & 459 & 100 \\
\hline \multirow{4}{*}{ Education } & University degree & 247 & 52.9 \\
\hline & Higher school/college degree & 180 & 38.5 \\
\hline & Other & 40 & 8.6 \\
\hline & Total & 467 & 100 \\
\hline \multirow{5}{*}{$\begin{array}{l}\text { Occupational } \\
\text { status/groups }\end{array}$} & Heads of units & 29 & 6.2 \\
\hline & Doctors & 132 & 28.3 \\
\hline & Nurses & 205 & 43.9 \\
\hline & Other health workers & 101 & 21.6 \\
\hline & Total & 467 & 100 \\
\hline \multirow{5}{*}{ Job seniority } & $\leq 3$ & 49 & 10.7 \\
\hline & $3.01-5$ & 24 & 5.2 \\
\hline & $5.01-10$ & 37 & 8.0 \\
\hline & $>10$ & 350 & 76.1 \\
\hline & Total & 460 & 100 \\
\hline
\end{tabular}

Tables 2-11 present the attitudes of the sociodemographic groups to the psychosocial risk determinants and organizational intervention objects (mean ranks, sample sizes (N), U values (the Mann-Whitney U-test) or $\chi 2$ values, with $k-1$ degrees of freedom,( the Kruskall-Wallis test) and significance levels (p)).

Gender. The observed differences by gender in the institution was not statistically significant except work-life balance. Women showed a higher average work-life balance score than men (Table 2; Table 3).

Age. Only excessive work pace as psychosocial risk determinant (Table 4) and justice of reward and matching to the job demands as organisational intervention objects (Table 5) had mean rank scores differing statistically across age groups. Excessive work pace increased consistently with age, with the oldest group of health workers $(>50)$ scoring highest on this factor. Justice of reward decreased consistently with age, with the youngest group of health workers $(\leq 30)$ scoring highest on this factor 
while the oldest group of health workers $(>50)$ gave a higher score for matching to the job demands than other age groups.

Job seniority. Excessive work pace as psychosocial risk determinant and justice of reward, matching to the job demands, and variety of tasks as organisational intervention objects had mean rank scores differing statistically across job seniority groups. Health workers who had more than 10 years tenure gave the highest scores for excessive work pace (Table 6), and health workers who had three to five years tenure gave the highest scores for all three organisational intervention objects (Table 7).

Table 2. Psychosocial risk determinants and gender results of the Mann-Whitney U-test.

\begin{tabular}{|c|c|c|c|c|c|c|}
\hline \multirow{2}{*}{ Variables } & \multicolumn{2}{|c|}{ Male } & \multicolumn{2}{|c|}{ Female } & \multirow{2}{*}{$u$} & \multirow{2}{*}{$p$} \\
\hline & Mean Rank & $N$ & Mean Rank & $N$ & & \\
\hline Hazardous Working Conditions & 259.38 & 24 & 232.63 & 443 & 4707.00 & 0.33 \\
\hline Work overload & 245.38 & 24 & 233.38 & 443 & 5043.00 & 0.66 \\
\hline Excessive work pace & 228.96 & 24 & 234.27 & 443 & 5195.00 & 0.84 \\
\hline Overtime & 222.73 & 24 & 234.61 & 443 & 5045.50 & 0.66 \\
\hline Tight deadlines & 250.25 & 24 & 233.12 & 443 & 4926.00 & 0.53 \\
\hline Unclear role & 227.79 & 24 & 234.34 & 443 & 5167.00 & 0.81 \\
\hline Conflicting roles & 260.98 & 24 & 232.54 & 443 & 4668.50 & 0.30 \\
\hline Being under-skilled for a job & 210.65 & 24 & 235.27 & 443 & 4755.50 & 0.37 \\
\hline Responsibility for decision making and actions & 250.25 & 24 & 233.12 & 443 & 4926.00 & 0.52 \\
\hline Lack of control over work pace & 211.17 & 24 & 235.24 & 443 & 4768.00 & 0.38 \\
\hline Lack of control over work method & 255.50 & 24 & 232.84 & 443 & 4800.00 & 0.40 \\
\hline Harassment & 240.42 & 24 & 233.65 & 443 & 5162.00 & 0.79 \\
\hline Conflicts & 253.29 & 24 & 232.95 & 443 & 4853.00 & 0.46 \\
\hline Tension & 241.08 & 24 & 233.62 & 443 & 5146.00 & 0.78 \\
\hline
\end{tabular}

Sample sizes-N, $U$ values - the Mann-Whitney U-test, $\chi^{2}$ values, with $\mathrm{k}-1$ degrees of freedom-the Kruskall-Wallis test, significance levels- $p$.

Table 3. Organizational intervention objects and gender results of the Mann-Whitney U-test.

\begin{tabular}{|c|c|c|c|c|c|c|}
\hline \multirow{2}{*}{ Variables } & \multicolumn{2}{|c|}{ Male } & \multicolumn{2}{|c|}{ Female } & \multirow{2}{*}{$u$} & \multirow{2}{*}{$p$} \\
\hline & Mean Rank & $N$ & Mean Rank & $N$ & & \\
\hline Work-Life Balance & 170.38 & 24 & 237.45 & 443 & 3789.00 & 0.01 \\
\hline Skills/abilities matching to the job demands & 204.90 & 24 & 235.58 & 443 & 4617.50 & 0.25 \\
\hline Variety of tasks & 247.58 & 24 & 233.26 & 443 & 4990.00 & 0.60 \\
\hline Social support & 215.81 & 24 & 234.99 & 443 & 4879.50 & 0.50 \\
\hline Organizational support & 208.90 & 24 & 235.36 & 443 & 4713.50 & 0.35 \\
\hline Participation in decision making & 198.54 & 24 & 235.92 & 443 & 4465.00 & 0.18 \\
\hline Communication & 198.02 & 24 & 235.95 & 443 & 4452.50 & 0.18 \\
\hline Justice of reward & 202.00 & 24 & 235.73 & 443 & 4548.00 & 0.23 \\
\hline Manager feedback & 230.58 & 24 & 234.19 & 443 & 5234.00 & 0.90 \\
\hline Stress management training & 230.63 & 24 & 234.18 & 443 & 5235.00 & 0.90 \\
\hline
\end{tabular}


Table 4. Psychosocial risk determinants and age group results of the Kruskall-Wallis test.

\begin{tabular}{|c|c|c|c|c|c|c|c|c|c|c|}
\hline \multirow{2}{*}{ Variables } & \multicolumn{2}{|l|}{$\leq 30$} & \multicolumn{2}{|l|}{ ]30-40] } & \multicolumn{2}{|l|}{ ]40-50] } & \multicolumn{2}{|c|}{$>50$} & \multirow{2}{*}{$X^{2}(3)$} & \multirow{2}{*}{$p$} \\
\hline & Mean Rank & $N$ & Mean Rank & $N$ & Mean Rank & $N$ & Mean Rank & $N$ & & \\
\hline Hazardous Working Conditions & 229.53 & 48 & 226.44 & 68 & 225.26 & 123 & 233.85 & 220 & 0.41 & 0.94 \\
\hline Work overload & 212.23 & 48 & 227.40 & 68 & 218.34 & 123 & 241.2 & 220 & 3.58 & 0.31 \\
\hline Excessive work pace & 177.81 & 48 & 207.74 & 68 & 227.13 & 123 & 249.87 & 220 & 17.36 & $<0.01$ \\
\hline Overtime & 199.69 & 48 & 229.10 & 68 & 233.84 & 123 & 234.75 & 220 & 3.21 & 0.36 \\
\hline Tight deadlines & 209.72 & 48 & 243.88 & 68 & 242.37 & 123 & 223.22 & 220 & 3.80 & 0.28 \\
\hline Unclear role & 198.13 & 48 & 217.11 & 68 & 224.30 & 123 & 244.13 & 220 & 6.63 & 0.08 \\
\hline Conflicting roles & 208.38 & 48 & 208.74 & 68 & 254.01 & 123 & 227.87 & 220 & 7.60 & 0.05 \\
\hline Being under-skilled for a job & 190.92 & 48 & 216.49 & 68 & 240.98 & 123 & 236.56 & 220 & 6.72 & 0.08 \\
\hline $\begin{array}{l}\text { Responsibility for decision } \\
\text { making and actions }\end{array}$ & 253.69 & 48 & 236.07 & 68 & 220.58 & 123 & 228.22 & 220 & 2.69 & 0.44 \\
\hline Lack of control over work pace & 277.73 & 48 & 232.26 & 68 & 222.39 & 123 & 223.14 & 220 & 7.63 & 0.05 \\
\hline Lack of control over work method & 247.51 & 48 & 259.38 & 68 & 226.01 & 123 & 219.33 & 220 & 6.27 & 0.10 \\
\hline Harassment & 231.72 & 48 & 233.09 & 68 & 246.96 & 123 & 219.19 & 220 & 4.30 & 0.23 \\
\hline Conflicts & 229.10 & 48 & 253.68 & 68 & 224.42 & 123 & 226.00 & 220 & 2.76 & 0.43 \\
\hline Tension & 228.82 & 48 & 246.32 & 68 & 224.31 & 123 & 228.39 & 220 & 1.40 & 0.71 \\
\hline
\end{tabular}

Table 5. Organizational intervention objects and age group results of the Kruskall-Wallis test.

\begin{tabular}{|c|c|c|c|c|c|c|c|c|c|c|}
\hline \multirow{2}{*}{ Variables } & \multicolumn{2}{|l|}{$\leq 30$} & \multicolumn{2}{|l|}{ ]30-40] } & \multicolumn{2}{|l|}{ ]40-50] } & \multicolumn{2}{|c|}{$>50$} & \multirow{2}{*}{$X^{2}(3)$} & \multirow{2}{*}{$p$} \\
\hline & Mean Rank & $N$ & Mean Rank & $N$ & Mean Rank & $N$ & Mean Rank & $N$ & & \\
\hline Work-Life Balance & 252.97 & 48 & 212.99 & 68 & 237.13 & 123 & 226.26 & 220 & 3.33 & 0.34 \\
\hline $\begin{array}{l}\text { Skills/abilities matching } \\
\text { to the job demands }\end{array}$ & 202.26 & 48 & 189.69 & 68 & 232.65 & 123 & 247.03 & 220 & 13.41 & $<0.01$ \\
\hline Variety of tasks & 232.03 & 48 & 222.53 & 68 & 231.51 & 123 & 231.02 & 220 & 0.27 & 0.96 \\
\hline Social support & 222.32 & 48 & 209.81 & 68 & 231.98 & 123 & 236.81 & 220 & 2.36 & 0.51 \\
\hline Organizational support & 243.05 & 48 & 223.24 & 68 & 234.60 & 123 & 226.67 & 220 & 0.94 & 0.81 \\
\hline Participation in decision making & 226.19 & 48 & 210.75 & 68 & 230.41 & 123 & 236.55 & 220 & 2.04 & 0.56 \\
\hline Communication & 230.20 & 48 & 201.90 & 68 & 225.49 & 123 & 241.16 & 220 & 4.80 & 0.19 \\
\hline Justice of reward & 278.61 & 48 & 247.19 & 68 & 235.06 & 123 & 211.25 & 220 & 12.44 & $<0.01$ \\
\hline Manager feedback & 223.95 & 48 & 228.51 & 68 & 230.33 & 123 & 231.60 & 220 & 0.14 & 0.99 \\
\hline Stress management training & 191.66 & 48 & 218.25 & 68 & 238.30 & 123 & 237.36 & 220 & 6.00 & 0.11 \\
\hline
\end{tabular}

Table 6. Psychosocial risk determinants and job seniority (working years) group results of the Kruskall-Wallis test.

\begin{tabular}{|c|c|c|c|c|c|c|c|c|c|c|}
\hline \multirow{2}{*}{ Variables } & \multicolumn{2}{|l|}{$\leq 3$} & \multicolumn{2}{|l|}{ ]3-5] } & \multicolumn{2}{|l|}{ ]5-10] } & \multicolumn{2}{|c|}{$>10$} & \multirow{2}{*}{$X^{2}(3)$} & \multirow{2}{*}{$p$} \\
\hline & Mean Rank & $N$ & Mean Rank & $N$ & Mean Rank & $N$ & Mean Rank & $N$ & & \\
\hline Hazardous Working Conditions & 209.59 & 49 & 262.04 & 24 & 225.24 & 37 & 231.82 & 350 & 2.78 & 0.43 \\
\hline Work overload & 221.77 & 49 & 206.17 & 24 & 215.36 & 37 & 234.99 & 350 & 1.99 & 0.57 \\
\hline Excessive work pace & 181.03 & 49 & 238.38 & 24 & 173.26 & 37 & 242.94 & 350 & 20.36 & $<0.01$ \\
\hline Overtime & 200.22 & 49 & 264.67 & 24 & 232.39 & 37 & 232.20 & 350 & 4.67 & 0.20 \\
\hline Tight deadlines & 223.78 & 49 & 248.33 & 24 & 206.72 & 37 & 232.73 & 350 & 2.00 & 0.57 \\
\hline Unclear role & 214.83 & 49 & 189.77 & 24 & 210.69 & 37 & 237.58 & 350 & 5.14 & 0.16 \\
\hline Conflicting roles & 201.95 & 49 & 253.58 & 24 & 213.88 & 37 & 234.67 & 350 & 4.17 & 0.24 \\
\hline Being under-skilled for a job & 201.15 & 49 & 265.5 & 24 & 207.45 & 37 & 234.65 & 350 & 5.92 & 0.12 \\
\hline $\begin{array}{l}\text { Responsibility for decision making } \\
\text { and actions }\end{array}$ & 245.51 & 49 & 238.48 & 24 & 227.64 & 37 & 228.15 & 350 & 0.97 & 0.81 \\
\hline Lack of control over work pace & 266.15 & 49 & 185.44 & 24 & 206.46 & 37 & 231.14 & 350 & 7.92 & 0.05 \\
\hline Lack of control over work method & 235.12 & 49 & 244.90 & 24 & 215.16 & 37 & 230.49 & 350 & 0.92 & 0.82 \\
\hline Harassment & 230.80 & 49 & 236.67 & 24 & 255.22 & 37 & 227.42 & 350 & 1.86 & 0.60 \\
\hline Conflicts & 215.26 & 49 & 272.52 & 24 & 251.55 & 37 & 227.53 & 350 & 4.42 & 0.22 \\
\hline Tension & 229.86 & 49 & 240.65 & 24 & 228.07 & 37 & 230.15 & 350 & 0.17 & 0.98 \\
\hline
\end{tabular}


Table 7. Organizational intervention objects and job seniority (working years) group results of the Kruskall-Wallis test.

\begin{tabular}{|c|c|c|c|c|c|c|c|c|c|c|}
\hline \multirow{2}{*}{ Variables } & \multicolumn{2}{|l|}{$\leq 3$} & \multicolumn{2}{|l|}{ ]3-5] } & \multicolumn{2}{|l|}{ ]5-10] } & \multicolumn{2}{|c|}{$>10$} & \multirow{2}{*}{$X^{2}(3)$} & \multirow{2}{*}{$p$} \\
\hline & Mean Rank & $N$ & Mean Rank & $N$ & Mean Rank & $N$ & Mean Rank & $N$ & & \\
\hline Work-Life Balance & 238.47 & 49 & 259.69 & 24 & 212.14 & 37 & 229.32 & 350 & 2.23 & 0.53 \\
\hline $\begin{array}{l}\text { Skills/abilities matching to the job } \\
\text { demands }\end{array}$ & 181.48 & 49 & 255.65 & 24 & 194.91 & 37 & 239.40 & 350 & 13.07 & $<0.01$ \\
\hline Variety of tasks & 220.63 & 49 & 323.31 & 24 & 228.92 & 37 & 225.68 & 350 & 12.98 & $<0.01$ \\
\hline Social support & 227.55 & 49 & 255.31 & 24 & 219.93 & 37 & 230.33 & 350 & 1.10 & 0.78 \\
\hline Organizational support & 241.46 & 49 & 257.73 & 24 & 241.74 & 37 & 225.91 & 350 & 2.04 & 0.56 \\
\hline Participation in decision making & 215.94 & 49 & 269.9 & 24 & 217.54 & 37 & 231.21 & 350 & 3.10 & 0.38 \\
\hline Communication & 227.79 & 49 & 240.60 & 24 & 201.36 & 37 & 233.27 & 350 & 2.11 & 0.55 \\
\hline Justice of reward & 288.12 & 49 & 290.73 & 24 & 268.65 & 37 & 214.27 & 350 & 22.91 & $<0.01$ \\
\hline Manager feedback & 212.77 & 49 & 280.75 & 24 & 230.74 & 37 & 229.51 & 350 & 4.34 & 0.23 \\
\hline Stress management training & 191.08 & 49 & 241.29 & 24 & 230.18 & 37 & 235.31 & 350 & 5.18 & 0.16 \\
\hline
\end{tabular}

Education. Four psychosocial risk determinants (overtime, unclear role, conflicting roles, and being under-skilled) and five organisational intervention objects (work-life balance, variety of tasks, communication, manager feedback, and stress management training) had mean rank scores differing statistically across education groups. Health workers who hold university degrees gave the highest scores for overtime, health workers who hold higher school degrees gave the highest scores for being under-skilled, health workers with other levels of education gave the highest scores for unclear role and conflicting roles (Table 8). Mean rank results also showed that a variety of tasks, communication, manager feedback, and stress management training were more highly valued by health workers with other levels of education and that work-life balance was more appreciated by health workers who hold higher school degrees (Table 9).

Table 8. Psychosocial risk determinants and education group results of the Kruskall-Wallis test.

\begin{tabular}{|c|c|c|c|c|c|c|c|c|}
\hline \multirow{2}{*}{ Variables } & \multicolumn{2}{|c|}{ University } & \multicolumn{2}{|c|}{ Higher School } & \multicolumn{2}{|l|}{ Other } & \multirow{2}{*}{$X^{2}(2)$} & \multirow{2}{*}{$p$} \\
\hline & Mean Rank & $N$ & Mean Rank & $N$ & Mean Rank & $N$ & & \\
\hline Hazardous Working Conditions & 229.97 & 247 & 238.26 & 180 & 239.71 & 40 & 0.49 & 0.78 \\
\hline Work overload & 235.40 & 247 & 235.93 & 180 & 216.69 & 40 & 0.76 & 0.68 \\
\hline Excessive work pace & 222.64 & 247 & 251.31 & 180 & 226.26 & 40 & 5.88 & 0.05 \\
\hline Overtime & 249.66 & 247 & 215.19 & 180 & 221.95 & 40 & 7.93 & 0.02 \\
\hline Tight deadlines & 234.83 & 247 & 235.31 & 180 & 223.00 & 40 & 0.32 & 0.85 \\
\hline Unclear role & 207.93 & 247 & 262.09 & 180 & 268.59 & 40 & 21.26 & $<0.01$ \\
\hline Conflicting roles & 220.63 & 247 & 241.75 & 180 & 281.68 & 40 & 8.56 & 0.01 \\
\hline Being under-skilled for a job & 208.45 & 247 & 263.37 & 180 & 259.63 & 40 & 20.22 & $<0.01$ \\
\hline $\begin{array}{l}\text { Responsibility for decision } \\
\text { making and actions }\end{array}$ & 242.40 & 247 & 228.50 & 180 & 206.85 & 40 & 3.32 & 0.19 \\
\hline Lack of control over work pace & 244.37 & 247 & 221.86 & 180 & 224.63 & 40 & 3.28 & 0.19 \\
\hline Lack of control over work method & 242.11 & 247 & 225.11 & 180 & 223.95 & 40 & 2.08 & 0.35 \\
\hline Harassment & 226.46 & 247 & 237.20 & 180 & 266.13 & 40 & 3.84 & 0.15 \\
\hline Conflicts & 230.51 & 247 & 237.87 & 180 & 238.10 & 40 & 0.37 & 0.83 \\
\hline Tension & 233.95 & 247 & 233.01 & 180 & 238.78 & 40 & 0.06 & 0.97 \\
\hline
\end{tabular}

Occupational groups. Six psychosocial risk determinants (work overload, overtime, tight deadlines, unclear role, being under-skilled, and responsibility) and all organisational intervention objects (except stress management training) had mean rank scores differing statistically across occupational groups. Doctors reported the most negative perception of workload, overtime, and tight deadlines; nurses notified the most negative perception of being under-skilled; other health workers declared the most negative perception of unclear role; and heads of units marked the most negative perception of responsibility (Table 10). The highest scores almost for all organisational intervention objects was given by heads of units, and that only for variety of tasks was given by other health workers (Table 11). 
Table 9. Organizational intervention objects and education group results of the Kruskall-Wallis test.

\begin{tabular}{cccccccccc}
\hline \multirow{2}{*}{ Variables } & \multicolumn{2}{c}{ University } & \multicolumn{2}{c}{ Higher school } & Other & \multirow{2}{*}{$\boldsymbol{X}^{\mathbf{2}(\mathbf{2})}$} & \multirow{2}{*}{$\boldsymbol{p}$} \\
\cline { 2 - 7 } & Mean Rank & $\boldsymbol{N}$ & Mean Rank & $\boldsymbol{N}$ & Mean Rank & $\boldsymbol{N}$ & & \\
\hline Work-Life Balance & 223.88 & 247 & 253.88 & 180 & 207.00 & 40 & 7.44 & 0.02 \\
\hline $\begin{array}{c}\text { Skills/abilities matching } \\
\text { to the job demands }\end{array}$ & 229.74 & 247 & 239.09 & 180 & 237.40 & 40 & 0.59 & 0.74 \\
\hline Variety of tasks & 208.24 & 247 & 257.81 & 180 & 285.91 & 40 & 21.42 & $<0.01$ \\
\hline Social support & 226.35 & 247 & 238.62 & 180 & 260.44 & 40 & 2.56 & 0.28 \\
\hline Organizational support & 229.88 & 247 & 235.19 & 180 & 254.09 & 40 & 1.14 & 0.56 \\
\hline Participation in decision making & 224.28 & 247 & 239.38 & 180 & 269.85 & 40 & 4.45 & 0.11 \\
\hline Communication & 217.23 & 247 & 247.41 & 180 & 277.24 & 40 & 9.80 & $<0.01$ \\
\hline Justice of reward & 233.40 & 247 & 229.30 & 180 & 258.84 & 40 & 1.61 & 0.45 \\
\hline Manager feedback & 227.04 & 247 & 228.60 & 180 & 301.30 & 40 & 10.95 & $<0.01$ \\
\hline Stress management training & 211.19 & 247 & 254.09 & 180 & 284.45 & 40 & 17.49 & $<0.01$ \\
\hline
\end{tabular}

Table 10. Psychosocial risk determinants and occupational group results of the Kruskall-Wallis test.

\begin{tabular}{|c|c|c|c|c|c|c|c|c|c|c|}
\hline \multirow{2}{*}{ Variables } & \multicolumn{2}{|c|}{ Heads of the Units } & \multicolumn{2}{|l|}{ Doctors } & \multicolumn{2}{|l|}{ Nurses } & \multicolumn{2}{|c|}{ Other } & \multirow{2}{*}{$X^{2}(3)$} & \multirow{2}{*}{$p$} \\
\hline & Mean Rank & $N$ & Mean Rank & $N$ & Mean Rank & $N$ & Mean Rank & $N$ & & \\
\hline Hazardous Working Conditions & 169.83 & 29 & 235.16 & 132 & 236.41 & 205 & 246.01 & 101 & 7.79 & 0.05 \\
\hline Excessive work pace & 230.62 & 29 & 242.72 & 132 & 232.08 & 205 & 227.47 & 101 & 1.03 & 0.79 \\
\hline Overtime & 245.28 & 29 & 263.42 & 132 & 229.85 & 205 & 200.73 & 101 & 14.23 & $<0.01$ \\
\hline Conflicting roles & 219.72 & 29 & 224.91 & 132 & 228.67 & 205 & 260.80 & 101 & 5.58 & 0.13 \\
\hline Being under-skilled for a job & 193.55 & 29 & 212.52 & 132 & 251.81 & 205 & 237.53 & 101 & 10.30 & 0.02 \\
\hline $\begin{array}{l}\text { Responsibility for decision } \\
\text { making and actions }\end{array}$ & 282.62 & 29 & 252.87 & 132 & 230.21 & 205 & 203.07 & 101 & 13.66 & $<0.01$ \\
\hline Conflicts & 222.72 & 29 & 220.33 & 132 & 249.84 & 205 & 222.95 & 101 & 5.39 & 0.14 \\
\hline Tension & 207.55 & 29 & 222.98 & 132 & 246.24 & 205 & 231.16 & 101 & 4.03 & 0.26 \\
\hline
\end{tabular}

Table 11. Organizational intervention objects and occupational group results of the Kruskall-Wallis test.

\begin{tabular}{ccccccccccc}
\hline \multirow{2}{*}{ Variables } & \multicolumn{1}{c}{ Heads of the Units } & \multicolumn{2}{c}{ Doctors } & \multicolumn{2}{c}{ Nurses } & \multicolumn{2}{c}{ Other } & \multirow{2}{*}{$\boldsymbol{X}^{\mathbf{2}(3)}$} & \multirow{2}{*}{$\boldsymbol{p}$} \\
\cline { 2 - 11 } & Mean Rank & $\boldsymbol{N}$ & Mean Rank & $\boldsymbol{N}$ & Mean Rank & $\boldsymbol{N}$ & Mean Rank & $\boldsymbol{N}$ & & \\
\hline Work-Life Balance & 282.10 & 29 & 202.67 & 132 & 244.51 & 205 & 239.81 & 101 & 13.19 & $<0.01$ \\
\hline $\begin{array}{c}\text { Skills/abilities matching } \\
\text { to the job demands }\end{array}$ & 295.91 & 29 & 240.48 & 132 & 238.66 & 205 & 198.30 & 101 & 15.29 & $<0.01$ \\
\hline Variety of tasks & 152.57 & 29 & 203.43 & 132 & 228.24 & 205 & 309.02 & 101 & 51.06 & $<0.01$ \\
\hline Social support & 295.64 & 29 & 213.57 & 132 & 236.10 & 205 & 238.73 & 101 & 9.33 & 0.02 \\
\hline Organizational support & 332.00 & 29 & 218.53 & 132 & 235.45 & 205 & 223.12 & 101 & 17.88 & $<0.01$ \\
\hline Participation in decision making & 295.64 & 29 & 217.84 & 132 & 235.33 & 205 & 234.72 & 101 & 8.08 & 0.04 \\
\hline Communication & 270.62 & 29 & 204.50 & 132 & 241.47 & 205 & 246.87 & 101 & 10.10 & 0.02 \\
\hline Justice of reward & 292.10 & 29 & 207.33 & 132 & 230.25 & 205 & 259.78 & 101 & 14.70 & $<0.01$ \\
\hline Manager feedback & 308.00 & 29 & 215.63 & 132 & 223.57 & 205 & 257.94 & 101 & 15.65 & $<0.01$ \\
\hline Stress management training & 235.90 & 29 & 214.92 & 132 & 244.20 & 205 & 237.68 & 101 & 4,09 & 0.25 \\
\hline
\end{tabular}

\section{Discussion}

The study aimed to explore whether psychosocial risk determinants and organizational intervention objects differ across employees' groups based on sociodemographic factors in a public primary healthcare institution. The findings of the study confirmed that different sociodemographic groups of health workers emphasized different psychosocial risk determinants and organisational intervention objects. 
In fact, there was no difference between gender groups. Only one organisational intervention object, work-life balance, was more relevant to women than to men. This may be due to gender imbalances in the institution-as is the case in all Lithuanian primary healthcare institutions. However, previous research findings provide contradictory information on gender differences in wellbeing at work [21]. The study asserted that only one psychosocial risk determinant-excessive work pace, which has led to negative impact-increased consistently with age, contrary to previous research [22]. Regarding organizational interventions objects and age groups, the youngest group of health workers $(\leq 30)$ emphasized more justice of reward, while the oldest group of health workers $(>50)$ valued more matching to the job demands. A possible explanation for this might be that age might affect several components of the stress process at work; as these effects are partly conflicting, they might nullify each other in the overall relation between age and stress [23]. Only one psychosocial risk determinant and three organisational intervention objects were statistically significantly different among job seniority groups. Once more, excessive work pace had the greatest negative impact on health workers with the highest seniority $(>10)$. Justice of reward, variety of tasks, and matching to the job demands were the most relevant to health workers who worked three to five years in the institution. These findings are original, but it can be due to specific standards and regulations in the Lithuanian public primary healthcare sector [24]. Compared with other groups, educational and professional groups significantly differed in their approach to psychosocial risk determinants and organizational intervention objects. Health workers who hold university degrees emphasized overtime, while health workers who hold higher school degrees stressed being under-skilled and health workers with other levels of education pointed out unclear role and conflicting roles. This is in contrast to Marinaccio' findings [25] that showed that workers with highest level of education perceived more role ambiguity and had skills that exceeds their job requirements. Interpretation may be that these results were influenced by specificity of work in the public healthcare institution. Regarding organisational intervention objects, work-life balance was the most relevant to health workers who hold higher school degrees. Variety of tasks, communication, manager feedback, and stress management training were the most relevant to health workers with other levels of education, confirming that "having little chance of formal promotion prospect for professional acknowledgement and respect lie in building informal competence and local reputation" [25]. Furthermore, more than half of the psychosocial risk determinants significantly differed due to occupational groups. Responsibility for decision making and actions was the most psychosocial risk determinant per the perception of heads of units. Doctors stressed workload, overtime, and tight deadlines, while nurses emphasized being under-skilled and other health workers pointed out unclear roles. This may be considered as specificity of the public healthcare institution. Heads' of units and doctors' tasks may fit their knowledge, skills, and abilities needed to perform the job; nurses' and others health workers' tasks may go beyond the job descriptions. These results are in line with previous studies suggesting that nurses have a coordinating role, the responsibility for shuttling between professional, organisational, and relational tasks [26]. Surprisingly, all organisational intervention objects were the most relevant to the heads of units, except that variety of tasks was the most relevant to other health workers, while doctors perceived almost all organisational intervention objects (social support, organisational support, participation in decision making, communication, justice of reward, and manager feedback) as irrelevant. In line with the literature, the findings confirm that public sector doctors' work is busier and more stressful than other health workers' work, and this may lead to psychological problems and burnout [21,27,28].

In summary, the results are in accordance with the studies indicating that occupational groups are the key factor that should be considered when managing psychosocial risks at organisation [12,29-31]. Other sociodemographic groups such as gender, age, education, and job seniority should be considered, with caution as an additional information because studies were very controversial on how and whom they influence [22].

Nevertheless, this study also has limitations. First, the study is a cross-sectional study and cannot make conclusions regarding causality. Second, the findings of this study are restricted to the public 
healthcare institution. Third, it did not include individual intervention objects that focus on helping individual employees to develop skills to manage, cope with, and reduce stress at work, whereas organisation-level interventions address the health and wellbeing of relatively large groups of workers in a uniform way [32]. Fourth, the small number of male workers in the sample may affect other subgroups of health workers (e.g., heads of units or some categories of job seniority), but it is the case for many Lithuanian primary healthcare institutions. Notwithstanding its limitations, this study provides some support for an integrated approach to the consideration of target groups in order to diagnose psychosocial risk determinants and to tailor organisational interventions to their specific needs at the institution. Healthcare institutions are likely to comprise competing and overlapping professional groups, and a key challenge is to consider carefully the impact of change on specific groups (e.g. doctors, nurses, and other health professionals and managers) and to design appropriate policies $[28,33]$.

\section{Conclusions}

The findings suggest that systematic assessment of risk groups on the basis of sociodemographic factors, especially occupational status, could facilitate psychosocial risk management in the public primary healthcare institution. It should be noted that the results of this study were based on a specific sample and that the method that was used to make internal comparisons of attitudes of different sociodemographic groups towards the psychosocial risk management inherently challenges generalizability and replicability of the results. Nevertheless, the findings might be generalized to countries with tax-financed universal healthcare system, considering the sociocultural context.

The study has proposed a way of diagnosing psychosocial risks and of tailoring interventions to all health workers of the public primary healthcare institution by using a simple and robust tool. For future research, it would be useful to examine attitudes of different sociodemographic groups towards the psychosocial risks and organizational intervention objects in a private primary healthcare institution. In addition, the study and the findings can be a basis for developing stress management programs in public healthcare institutions and an incentive for new theories of qualitative studies.

Author Contributions: Conceptualization, D.D. and R.S.; methodology, D.D.; validation, D.D., R.S., and A.J.-R.; formal analysis, D.D.; investigation, D.D.; resources, A.J.-R.; data curation, D.D. writing-original draft preparation, D.D.; writing-review and editing, D.D., R.S., and A.J.-R.; visualization, D.D.; supervision, R.S.; project administration, D.D. All authors have read and agreed to the published version of the manuscript.

Funding: This research received no external funding.

Conflicts of Interest: The authors declare no conflict of interest.

\section{References}

1. Ford, M.T. Job-Occupation Misfit as an Occupational Stressor. J. Vocat. Behav. 2012, 80, 412-421. [CrossRef]

2. Gilboa, S.; Shirom, A.; Fried, Y.; Cooper, C. A meta-analysis of work demand stressors and job performance: Examining main and moderating effects. Pers. Psychol. 2008, 61, 227-271. [CrossRef]

3. Lopes, C.S.; Araya, R.; Werneck, G.L.; Chor, D.; Faerstein, E. Job strain and other work conditions: Relationships with psychological distress among civil servants in Rio de Janeiro, Brazil. Soc. Psychiat. Epidemiol. 2010, 45, 345-354. [CrossRef] [PubMed]

4. Melchior, M.; Krieger, N.; Kawachi, I.; Berkman, L.F; Niedhammer, I.; Goldberg, M. Work factors and occupational class disparities in sickness absence: Findings from the GAZEL cohort study. Am. J. Public Health 2005, 95, 1206-1212. [CrossRef]

5. Council Directive 89/391/EEC of 12 June 1989 on the Introduction of Measures to Encourage Improvements in the Safety and Health of Workers at Work. Available online: http://data.europa.eu/eli/dir/1989/391/2008-12-11 (accessed on 6 December 1989).

6. Lamontagne, A.D.; Keegel, T.; Louie, A.M.; Ostry, A.; Landsbergis, P.A. A systematic review of the job-stress intervention evaluation literature, 1990-2005. Int. J. Occup. Environ. Health 2007, 13, 268-280. [CrossRef] 
7. Bourbonnais, R.; Brisson, C.; Vinet, A.; Vézina, M.; Abdous, B.; Gaudet, M. Effectiveness of a participative intervention on psychosocial work factors to prevent mental health problems in a hospital setting. Occup. Environ. Med. 2006, 63, 335-342. [CrossRef]

8. Milner, A.; Butterworth, P.; Bentley, R.; Kavanagh, A.M.; LaMontagne, A.D. Sickness Absence and Psychosocial Job Quality: An Analysis from a Longitudinal Survey of Working Australians, 2005-2012. Am. J. Epidemiol. 2015, 181, 781-788. [CrossRef]

9. Egan, M.; Bambra, C.; Thomas, S.; Petticrew, M.; Whitehead, M.; Thomson, H. The psychosocial and health effects of workplace reorganisation. 1. A systematic review of organisational-level interventions that aim to increase employee control. J. Epidemiol. Commun. Health 2007, 61, 945-954. [CrossRef]

10. Nielsen, K.; Fredslund, H.; Christensen, K.B.; Albertsen, K. Success or failure? Interpreting and understanding the impact of interventions in four similar worksites. J. Work Stress 2006, 20, 272-287. [CrossRef]

11. Aust, B.; Rugulies, R.; Finken, A.; Jensen, C. When workplace interventions lead to negative effects: Learning from failures. Scand. J. Public Health 2010, 38, 106-119. [CrossRef]

12. Chan, K.B.; Lai, G.; Ko, Y.C.; Boey, K.W. Work stress among six professional groups: The Singapore experience. J. Soc. Sci. Med. 2000, 50, 1415-1432. [CrossRef]

13. Brewer, E.W.; Shapard, L. Employee burnout: A meta-analysis of the relationship between age or years of experience. Hum. Resour. Dev. Rev. 2004, 3, 102-123. [CrossRef]

14. Kompier, M.A.J.; Cooper, C.L.; Geurts, S.A.E. A multiple case study approach to work stress prevention in Europe. Eur. J. Work Organ. Psychol. 2000, 9, 371-400. [CrossRef]

15. Happell, B.; Dwyer, T.; Reid-Searl, K.; Burke, A.K.J.; Caperchione, C.M.; Gaskin, C. Nurses and stress: Recognizing causes and seeking solutions. J. Nurs. Manag. 2013, 21, 638-647. [CrossRef] [PubMed]

16. Randall, R.; Nielsen, K.; Tvedt, S.D. The development of five scales to measure employees' appraisals of organizational-level stress management interventions. J. Work Stress 2009, 23, 1-23. [CrossRef]

17. Kristof-Brown, A.L.; Zimmerman, R.D.; Johnson, E.C. Consequences of individuals' fit at work: A meta-analysis of person-job, person-organization, person-group, and person-supervisor fit. J. Pers. Psychol. 2005, 58, 281-342. [CrossRef]

18. Randall, R.; Cox, T.; Griffiths, A. Participants' accounts of a stress management intervention. J. Hum. Relat. 2007, 60, 1181-1209. [CrossRef]

19. Mäkikangasa, A.; Kinnunenb, U.; Feldta, T.; Schaufelic, W. The longitudinal development of employee well-being: A systematic review. J. Work Stress 2016, 30, 46-70. [CrossRef]

20. Bandzienė, A. Kompleksinis Streso Darbe Valdymas (Complex Stress Management at Work). Ph.D. Thesis, ISM Vadybos ir Ekonomikos Universitetas, Kaunas, Lithuania, 2009.

21. Vanagas, G.; Bihari-Axelsson, S.; Vanagienè, V. Do age, gender and marital status influence job strain development for general practitioner? Medicina 2004, 40, 1014-1018.

22. Kulvinskienè, V.R.; Bandzienè, A. Streso darbe ekonominis kontekstas (Economical context of work-related stress). Ekonomika 2008, 82, 75-90.

23. Rauschenbach, C.; Krumm, S.; Thielgen, M.; Hertel, G. Age and work-related stress: A review and meta-analysis. J. Manag. Psychol. 2013, 28, 781-804. [CrossRef]

24. The Lithuanian National Health System Sector Collective Agreement. Available online: https://sam.lrv.lt/lt/ teisine-informacija/sakos-kolektyvines-sutartys (accessed on 6 December 2018).

25. Marinaccio, A.; Ferrante, P.; Corfiati, M.; Tecco, C.; Rondinone, B.M.; Bonafede, M.; Ronchetti, M.; Persechino, B.; Iavicoli, S. The relevance of socio-demographic and occupational variables for the assessment of work-related stress risk. BMC Public Health 2013, 13, 1157-1165. [CrossRef] [PubMed]

26. Krogstad, U.; Hofoss, D.; Veenstra, M.; Hjortdahl, P. Predictors of job satisfaction among doctors, nurses and auxiliaries in Norwegian hospitals: Relevance for micro unit culture. Hum. Resour. Health 2006, 4, 3-10. [CrossRef] [PubMed]

27. Heponiemi, T.; Kouvonen, A.; Aalto, A.M.; Elovainio, M. Psychosocial factors in GP work: The effects of taking a GP position or leaving GP work. Eur. J. Public Health 2012, 23, 361-366. [CrossRef]

28. Nikolic, D.; Višnjic, A. Mobbing and Violence at Work as Hidden Stressors and Work Ability among Emergency Medical Doctors in Serbia. Medicina 2020, 56, 31. [CrossRef] [PubMed]

29. De Smet, P.; Sans, S.; Dramaix, M.; Boulenguez, C.; De Backer, G.; Ferrario, M.M.; Cesana, G.; Houtman, I.; Isacsson, S.O.; Kittel, F.; et al. Gender and regional differences in perceived job stress across Europe. Eur. J. Public Health 2005, 15, 536-545. [CrossRef] 
30. Scott, T.; Mannion, R.; Davies, H.T.O.; Marshall, M.N. Implementing culture change in health care: Theory and practice. Int. J. Qual. Health Care 2003, 15, 111-118. [CrossRef]

31. Basu, S.; Harris, A.; Mason, S.; Norman, J. A longitudinal assessment of occupational stress in Emergency Department Nursing Staff. J. Nurs. Manag. 2019, 28, 167-174. [CrossRef]

32. Cox, T.; Taris, T.W.; Nielsen, K. Organizational interventions: Issues and challenges. Work Stress 2010, 24, 217-218. [CrossRef]

33. Niks, I.; de Jonge, J.; Gevers, J.; Houtman, I. Work Stress Interventions in Hospital Care: Effectiveness of the DISCovery Method. Int. J. Environ. Res. Public Health 2018, 15, 332. [CrossRef] 